good many coffee tables as well. Nevertheless, in my opinion, there is some fundamental intellectual dishonesty here, some snake oil being peddled. The authors are trying desperately to be taken seriously, and they do not always play fair with their readers.

While there is a lot of good scientific exposition in the book, there is too a distressing amount of what seems to be mathematical flim-flam, that is, quotation of precise results in a manner designed to mislead less-mathematical readers and cause them to jump to the authors' desired (usually non-mathematical) conclusion. For example, after some rather woolly discussion of Penrose conformal diagrams, Barrow and Tipler conclude "A Penrose diagram allows us to define rigorously 'an achieved infinity', a concept whose logical consistency philosophers have been doubtful about for thousands of years". This is a silly assertion, but it is put forth with the utmost gravity, in such a way that many readers will be taken in. And it is only one of many such cases.

Nor do the authors always play fair with other workers in their field. They have a tendency to bury footnote references to the work of their colleagues in and among a plethora of erudite footnotes to nineteenth- and twentieth-century precursors. This becomes particularly disturbing when, as is sometimes the case, the exposition mirrors other authors' papers practically sentence by sentence. The casual reader will come away mistakenly crediting Barrow and Tipler for ideas that they have, in fact, only nicely summarized. The serious scholar should take their bibliographical research and attributions (an immense amount of work on their part) as indicative, not definitive.

The book's flaws, I think, can be traced back to a single cause: the authors badly want to be the founding doctrinal theorists of a "new" resurgence of teleological belief in science. They bring to their cause an impressively broad knowledge of scientific exotica, but the factual content of the book is only their means to an end that (in my opinion) is threatening to the modern scientific enterprise. That end is nothing less than the fusion of matters of science with matters of individual faith and belief. It has taken us a long time to separate these matters, each to its own legitimate arena in human affairs. We should not lightly allow them to become once again jumbled, least of all by a book that now and then cuts corners in getting to its own pre-determined, distant goals. This is a fascinating and entertaining book, one to read and think about. But it is also one whose extra-scientific agenda most of us will, ultimately, wish to reject.

William H. Press is Professor of Astronomy and Physics at the Center for Astrophysics, Harvard University, 60 Garden Street, Cambridge, Mas sachusetts $02138, U S A$.

\section{What do intermediate filaments do?}

\section{Keith Burridge}

Intermediate Filaments: A Review. By Peter Traub. Springer-Verlag: 1985. Pp. 266. DM 168.

FOR SEVERAL years people interested in intermediate filaments have looked over their shoulders with envy at those studying microtubules and microfilaments. While there is ample evidence for the importance of microtubules and microfilaments in generating cellular movements, the function of intermediate filaments remains elusive. Because no obvious motility function has been apparent, some sort of skeletal or structural role has been generally, if begrudgingly, accepted, but this concept has not been embraced with the kind of enthusiasm that gives a field new momentum.

Anyone examining the function of intermediate filaments has some puzzling experimental observations to consider. Some cells exist, apparently quite happily, without them. Similarly, the intermediate filaments within a cell can be collapsed by microinjection of antibodies without detectable effects on the cell's behaviour. From these observations one might conclude the intermediate filaments are not very important, or, alternatively, that they have a subtle function which has been missed because the assays have been inappropriate. This latter argument is the one adopted by Peter Traub. He proposes that the idea that intermediate filaments have a structural role is essentially the consequence of depending on structural techniques, such as immunofluorescence and electron microscopy, to study them. Traub uses the final chapter of his book to present his own, novel ideas about possible functions; rather than being skeletal elements within cells, he suggests, intermediate filaments are involved in signal transduction from the plasma membrane or cytoplasm to the nucleus.

Traub first became entangled in this field accidentally. Working as a virologist, he became intrigued with a protein that was binding to some viral RNA. Only later did he realize that this protein had been discovered previously and was vimentin, the subunit of one of the main types of intermediate filament. At this point others might have dropped the subject, attributing the binding to irrelevant coincidence, but Traub and his colleagues have pursued the matter. They have shown under physiological conditions that the subunit proteins of several classes of intermediate filament have a specificity for binding to DNA rather than to RNA. From the work of others, it has been known for some time that intermediate filaments are very susceptible to proteolytic cleavage by a calcium-activated protease in the cytoplasm, and again Traub has demonstrated that the fragments generated from the different classes of filament by this protease retain the selective DNA-binding activity.

These biochemical observations form the basis of Traub's theory. He suggests that calcium elevated in the cytoplasm in response to external signals activates the protease. This, in turn, cleaves the intermediate filament subunits, which can no longer assemble, and which are transported back to the nucleus. Here they interact with DNA or chromatin, probably in conjunction with other proteins, in a regulatory manner. When I first read about this idea I was sceptical. I still am, but Traub assembles a considerable body of evidence that is consistent with the proposal. Certainly, an advantage of his hypothesis is that it suggests many experiments by which it could be tested. On the other hand, the idea that intermediate filaments have primarily a skeletal function is a difficult concept to establish or to destroy.

Only the last chapter of the book is dedicated to this unorthodox but refreshing concept. Regardless of whether or not one accepts it, the book as a whole provides an excellent review of intermediate filaments. Almost every aspect of the subject is covered, with references up until about 1984, ranging from heterogeneity, structure and assembly, to the interaction of intermediate filaments with other elements, secondary modification and response to normal and pathological stimuli. Given how vast and labyrinthine the field has become, this is quite an accomplishment. The only area in which Traub has not attempted to be comprehensive is the increasing clinical use of intermediate filaments for tumour typing.

The book should be useful both for specialists and for the many cell biologists who, like myself, have a peripheral interest in intermediate filaments. It is well organized, well indexed and has a bibliography of some 60 pages. I anticipate that it will be the standard work for many years. If it also stimulates some novel experiments that help to unravel the knot of intermediate filament function, then Traub should be well pleased with having provided a double service to the field.

Keith Burridge is in the Laboratories for Cell Biology, Department of Anatomy, University of North Carolina at Chapel Hill, 111 Swing Building 217H, Chapel Hill, North Carolina 27514, USA.

- Two recent books on the cytoskeleton are Molecular Biology of the Cytoskeleton (G.G. Borisy et al., eds), published by Cold Spring Harbor Laboratory, and The Cytoskeleton: An Introductory Survey (by M. Schliwa), published by Springer-Verlag. 